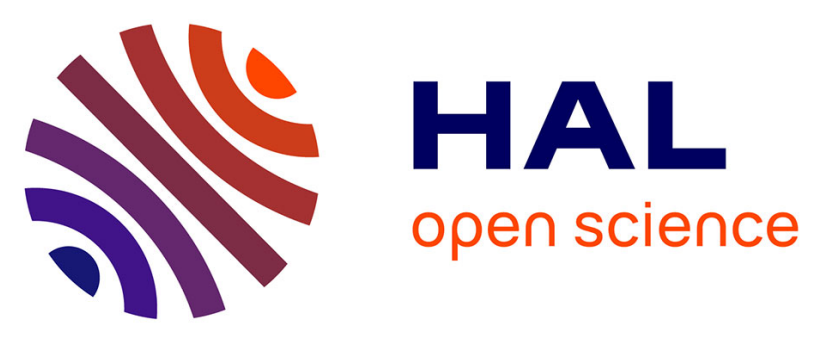

\title{
Applications of electron reduced density function analysis to the study of amorphous materials: an example of Zr64.13Cu15.75Ni10.12Al10.0 and Zr55Cu30Ni5Al10 metallic glasses
}

Konstantin Borisenko, Yixin Chen, Guoqiang Li

\section{To cite this version:}

Konstantin Borisenko, Yixin Chen, Guoqiang Li. Applications of electron reduced density function analysis to the study of amorphous materials: an example of Zr64.13Cu15.75Ni10.12Al10.0 and Zr55Cu30Ni5Al10 metallic glasses. Philosophical Magazine, 2010, pp.1. 10.1080/14786435.2010.487475 . hal-00603441

\section{HAL Id: hal-00603441 \\ https://hal.science/hal-00603441}

Submitted on 25 Jun 2011

HAL is a multi-disciplinary open access archive for the deposit and dissemination of scientific research documents, whether they are published or not. The documents may come from teaching and research institutions in France or abroad, or from public or private research centers.
L'archive ouverte pluridisciplinaire HAL, est destinée au dépôt et à la diffusion de documents scientifiques de niveau recherche, publiés ou non, émanant des établissements d'enseignement et de recherche français ou étrangers, des laboratoires publics ou privés. 


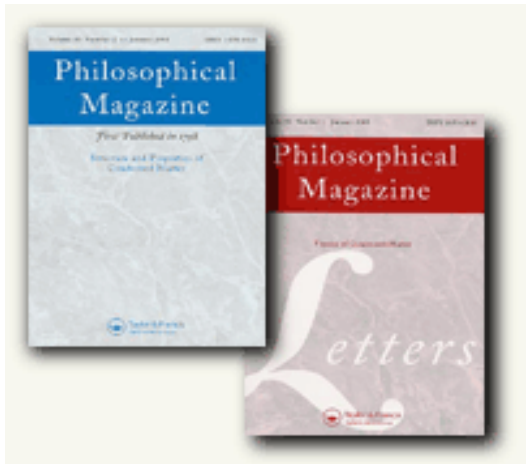

\section{Applications of electron reduced density function analysis to the study of amorphous materials: an example of Zr64.13Cu15.75Ni10.12Al10.0 and Zr55Cu30Ni5Al10 metallic glasses}

\begin{tabular}{|c|c|}
\hline Journal: & Philosophical Magazine \& Philosophical Magazine Letters \\
\hline Manuscript ID: & TPHM-09-Sep-0393.R1 \\
\hline Journal Selection: & Philosophical Magazine \\
\hline $\begin{array}{r}\text { Date Submitted by the } \\
\text { Author: }\end{array}$ & 12-Mar-2010 \\
\hline Complete List of Authors: & $\begin{array}{l}\text { Borisenko, Konstantin; University of Oxford, Department of } \\
\text { Materials } \\
\text { Chen, Yixin; University of Oxford } \\
\text { Li, Guoqiang; University of Oxford }\end{array}$ \\
\hline Keywords: & electron diffraction, metallic glasses, atomic structure, plasticity \\
\hline Keywords (user supplied): & \\
\hline
\end{tabular}

\section{(5) ScholaroNE \\ Manuscript Central}




\title{
Applications of electron reduced density function analysis to the study of amorphous materials: an example of $\mathrm{Zr}_{64.13} \mathrm{Cu}_{15.75} \mathrm{Ni}_{10.12} \mathrm{Al}_{10.0}$ and $\mathrm{Zr}_{55} \mathrm{Cu}_{30} \mathrm{Ni}_{5} \mathrm{Al}_{10}$ metallic glasses
}

\author{
Konstantin B. Borisenko, ${ }^{*}$ Yixin Chen and Guoqiang Li \\ Department of Materials, University of Oxford, Parks Road, Oxford, OX1 3PH, UK \\ Dedicated to Professor David J. H. Cockayne FRS
}

\begin{abstract}
Analysis of the reduced density functions from electron diffraction data and density functional theory calculations for the two metallic glasses with high plasticity with the compositions $\mathrm{Zr}_{64.13} \mathrm{Cu}_{15.75} \mathrm{Ni}_{10.12} \mathrm{Al}_{10.0}$ and $\mathrm{Zr}_{55} \mathrm{Cu}_{30} \mathrm{Ni}_{5} \mathrm{Al}_{10}$ has indicated that the structures are essentially randomly packed with small degree of local ordering. These structural features may be important in understanding the high plasticity of the glasses.
\end{abstract}

\section{Introduction}

Accurate reduced density functions (RDF) can now be routinely obtained from thin films of materials using the electron diffraction technique developed by Cockayne et al [1-3]. These functions are closely related to the radial distribution functions, taking into account the different 
scattering factors of different elements in the material. The technique provides unique information about distribution of interatomic distances in the material, on the basis of which atomistic models can be built and refined, and then related to the observed properties of the films.

The models can be refined using the observed experimental diffraction data in a Reverse Monte Carlo (RMC) routine [4,5] and also checked for minimum energy conditions using density functional theory (DFT) energy calculations. A combined refinement of the models with constraints obtained from DFT calculations leading to an agreement with both the experimental data and theoretical DFT models can also be applied.

One of the examples is in application to the study of atomic structures of metallic glasses. Metallic glasses have promising industrial potential due to their high strength and resistance to corrosion [6,7]. However, a serious limiting factor in their applications is their poor ductility. Recently, novel metallic glasses of general composition $\mathrm{Zr}-\mathrm{Cu}-\mathrm{Ni}$-Al with very good ductility have been prepared [8-10]. Such properties in metallic glasses are unusual and to understand them, obtaining reliable information on local atomic structure is very important.

There have been several recent structural studies of the related $\mathrm{Zr}-\mathrm{Cu}-\mathrm{Al}$ or $\mathrm{Zr}-\mathrm{Ni}-\mathrm{Al}$ metallic glasses by either experimental X-ray diffraction $[11,12]$ or theoretical techniques $[13,14]$; however additional and more reliable structural information can be obtained by combination of the two approaches.

In this paper, we describe application of the RDF technique using electron diffraction combined with the DFT calculations to study atomic structures of two metallic glasses with compositions $\mathrm{Zr}_{64.13} \mathrm{Cu}_{15.75} \mathrm{Ni}_{10.12} \mathrm{Al}_{10.0}$ and $\mathrm{Zr}_{55} \mathrm{Cu}_{30} \mathrm{Ni}_{5} \mathrm{Al}_{10}$.

\section{Electron diffraction experiment}


The metallic glasses with the nominal compositions (in at. \%) $\mathrm{Zr}_{64.13} \mathrm{Cu}_{15.75} \mathrm{Ni}_{10.12} \mathrm{Al}_{10.0}$ ( $\mathrm{Zr64CuNiAL})$ and $\mathrm{Zr}_{55} \mathrm{Cu}_{30} \mathrm{Ni}_{5} \mathrm{Al}_{10}(\mathrm{Zr} 55 \mathrm{CuNiAl})$ were prepared by arc melting the constituents under a Ti-gettered argon atmosphere in a water-cooled copper crucible. The alloys were cooled and remelted several times to ensure compositional homogeneity. In the final stages of the preparation they were cast into copper molds to form ingots of $3 \mathrm{~mm}$ diameter.

To prepare samples for transmission electron microscopy (TEM), the metallic glass ingots were first sliced into $3 \mathrm{~mm}$ discs, mechanically ground to a thickness of $\sim 40 \mu \mathrm{m}$, and then ionmilled in a precision ion polishing system (PIPS) operating at $3.0 \mathrm{kV}$ (Gatan, Pleasanton, CA) until appearance of a tiny hole in the sample. For final thinning the samples were moved to a low angle ion milling system with a liquid nitrogen cooling stage working at a low voltage of $300 \mathrm{~V}$ (Fischione 1010 Low Angle Ion Milling \& Polishing System) to prevent or remove any structural alteration possibly caused by the high-energy ion beam of PIPS. The electron diffraction experiments for both glasses were carried out on a JEOL 3000F field-emission gun TEM operating at $300 \mathrm{kV}$. Selected area diffraction (SAD) was used to collect the electron diffraction patterns from different thin areas in the samples. The selected area was, in each case, a disc of diameter about $100 \mathrm{~nm}$ on the sample. To avoid contribution from multiple scattering in the diffraction data, only very thin areas were used for SAD. The condition for the sample to be sufficiently thin to give only single scattering was that the total scattered intensity, $I(q)$, would oscillate about the sum of scattering intensities from independently scattering atoms, over the full range of $q$. Larger contribution of multiple scattering in thicker samples, to our experience, results in that a good fit can only be achieved in the $q$ range from usually 15 to $70 \mathrm{~nm}^{-1}$, while even adjusting the number of atoms as a fitting parameter will not produce a good fit in the higher $q$ 
range from 70 to $200 \mathrm{~nm}^{-1}$. The energy filtered TEM (EFTEM) elemental mapping has found no clustering of atoms and no crystallites were seen in the high resolution images of the materials. The diffraction patterns were recorded on a $1024 \times 1024$ pixel CCD camera and corrected for dark current and pixel sensitivity. All the diffraction patterns were accurately calibrated by using constant lens settings for the TEM and a polycrystalline gold sample assuring a better than $1 \%$ accuracy in interatomic distances. This corresponds to the error in all obtained distances of \pm 0.02 Å. Using a camera length of $15 \mathrm{~cm}$, diffraction patterns were collected up to a scattering vector of $q \simeq 220 \mathrm{~nm}^{-1}$, where $q=4 \pi \sin (\theta) / \lambda$. Here $\theta$ is the half scattering angle and $\lambda$ is the wavelength of electrons. The physical limit imposed by the size of the detector of about $2.5 \mathrm{~cm}$ would correspond to the maximum $q$ of about $530 \mathrm{~nm}^{-1}$. However, usable data for refinements extended up to $q=130 \mathrm{~nm}^{-1}$ due to low signal to noise ratio at higher $q$. From the obtained total scattering intensity, $I(q)$, the experimental reduced scattering intensity, $\phi(q)$, and then the reduced density function can be obtained as described in Refs. 1-3. As the studied materials are expected to contain no interatomic distances shorter than $2.0 \AA$, the contribution to the reduced scattering intensities that produced peaks in the RDF below $2.0 \AA$ was removed by a Fourier filter.

\section{Reverse Monte Carlo refinements}


The Reverse Monte Carlo refinements were performed by fitting the theoretical reduced scattering intensity computed from an atomistic model to that obtained experimentally as described above by minimizing the sum of the squared differences between the two data sets, $\Phi=\sum_{i}\left[\phi_{i}^{\exp }\left(q_{i}\right)-\phi_{i}^{\text {theor }}\left(q_{i}\right)\right]^{2}$. An additional cost function, $\mathrm{E}=\sum_{i}\left[r_{i}^{A B}-c_{i}^{A B}\right]^{2}$, related to the deviation of the first nearest neighbours in the model, $r_{i}^{A B}$, from values used as constraints, $c_{i}^{A B}$, for example, from the DFT calculations or the sum of the covalent radii of the elements, $c_{i}^{A B}=r_{A}^{\mathrm{cov}}+r_{B}^{\mathrm{cov}}$, was also introduced into the refinements. 
In this procedure, first, an atomistic model was constructed by random packing of atoms with periodic boundary conditions in three dimensions with the density close to the experimental density of similar studied materials, $6.6 \mathrm{~g} \mathrm{~cm}^{-3}$ [8]. From this model the theoretical reduced scattering intensity was computed utilizing the Debye approximation, $\phi(q)=\sum_{i} f_{i}(q)^{2}+\sum_{i} \sum_{j} \frac{\sin q r_{i j}}{q r_{i j}} B_{i j}$, where $B_{i j}=e^{-\frac{u_{i j}^{2} q^{2}}{2}}$ is the Debye-Waller type probabilitydensity factor to account for the phonon vibrations. The atomic scattering amplitudes, $f(q)$, compiled by Kirkland [15] were used. The vibrational amplitudes, $u_{i j}$, in the Debye-Waller factor were assumed to be $0.02 \AA$ and equal for all interatomic distances. The additional cost function $E$ was also evaluated. An atom was then selected randomly in the model and moved by a random amount within the specified distance from its initial position. The change in the reduced scattering intensity and its agreement with the corresponding experimental data as well as change in the cost function $E$ was then calculated. If the move improved the total agreement, expressed as $F=\sqrt{w_{\Phi} \Phi^{2}+w_{E} E^{2}}$, where $w_{\Phi}$ and $w_{E}$ are weights, it was accepted; if not, the move was accepted with the probability $e^{-\Delta F / M}$, where $M$ is a parameter. The typical value of $M$ used in the refinements was 0.002 . This procedure was repeated until a minimum in the total agreement, with good agreement in experimental data and low cost function values, was achieved.

\section{Density functional theory energy optimisations}


To prepare initial models for energy optimizations, models of 100 atoms were first refined using the described RMC procedure to agree with the experimental diffraction data. These RMCrefined models were then used as starting points for energy optimizations with full relaxation of the atomic positions within a fixed unit cell with periodic boundary conditions. The energy optimizations of the models were performed using the VASP code [16,17]. Projector-augmented wave potentials were employed for $\mathrm{Zr}, \mathrm{Cu}, \mathrm{Ni}$ and $\mathrm{Al}$ within the local density approximation (LDA) described by the Perdew and Zunger functional [18]. The plane-wave basis set energy cut off was $230 \mathrm{eV}$. The relaxation of the structures continued until the change in energy for ions was less than $1 \times 10^{-5} \mathrm{eV}$. The convergence criterion for maximum force on ions was $-0.01 \mathrm{eV} / \AA$. An $2 \times 2 \times 2$ grid of k points, automatically generated by the Monkhorst and Pack algorithm [19], was used for sampling of the electron density. This scheme produced k-points with their origin offset from the gamma point by $1 / 4$.

\section{Results and discussion}

Comparison of the experimental RDFs obtained for the two metallic glasses is presented in Figure 1. It is seen that changing the composition of the glass results in a prominent shift of the first three peaks in the RDFs to shorter distances. The main experimental peak positions for the two glasses are summarized in Table 1. 
The RDFs obtained from the models from the DFT calculations for the two metallic glasses show qualitative agreement with the experimental RDFs in the number and shape of the peaks, Figure 2, although the bond lengths in the energy-optimised models contributing to the first peak are shorter, resulting in a shift of the peak to shorter values. The peaks of the RDFs obtained from the energy-optimized models are shown in Table 1. The difference in position between the observed and computed peaks is about $4 \%$, which can be regarded as satisfactory. The experimentally observed trend of shortening first neighbour distances when going from $\mathrm{Zr64CuNiAl}$ to $\mathrm{Zr55CuNiAl}$ metallic glass is well reproduced in the energy-optimized models.

Using the average bond length obtained in the DFT calculations of the models of the metallic glasses as constraints, atomic models were prepared by random packing of 1000 atoms. The RDFs computed from these models are compared to the experimental ones in Figure 3.

Although the randomly packed models do not agree quantitatively with the experimental RDF curves, their shapes appear to be similar. This suggests that there is certain amount of local ordering in the materials, although it is not particularly strong and the structure resembles a randomly packed model to a considerable degree.

Using the same bond-length constraints obtained from the DFT calculations, the models of the metallic glasses were refined against the experimental data in the RMC procedure. For these refinements the weights for the experimental data and theoretical constraints were selected to obtain a compromise model where the constraints are satisfied as much as possible while maintaining a good fit to the experimental diffraction data. The resulting RDFs from the refined models are compared to the experimental ones in Figure 4. 
Using the constraints for bond lengths from the DFT calculations has resulted in a somewhat worse fit in the first peak of the RDFs. The average bond lengths and coordination numbers from the RMC refinements together with those from the DFT calculations are shown in Table 2 .

The structures of both glasses have similar average bond lengths, some of which are considerably different from the corresponding bond lengths from the DFT calculations, as they had to be adjusted to fit the experimental diffraction data. While $\mathrm{Zr}-\mathrm{Zr}$ bond lengths agree relatively well, there is, for example, considerable lengthening of the $\mathrm{Zr}-\mathrm{Cu}$ bond lengths in the refined structure as compared to that from the DFT energy optimizations, Table 2. In addition, the absolute values of the coordination numbers from the two techniques differ considerably, although the general trends in the change of the coordination number when going from the $\mathrm{Zr64CuNiAl}$ to $\mathrm{Zr} 55 \mathrm{CuNiAl}$ metallic glass do agree. For instance, the coordination number of $\mathrm{Zr}$, $N_{\mathrm{Zr}}$, is the same in the two metallic glasses, while that of the copper, $N_{\mathrm{Cu}}$, increases.

Analysing the structures from the DFT energy calculations and RMC refinements, it is seen that the energy optimisations lead to small but noticeable changes in the structures, reflected in bond type, bond angle and dihedral angle distribution histograms. In the energy-optimised models of both glasses a decrease in the probability of finding $\mathrm{Zr}-\mathrm{Zr}$ and $\mathrm{Cu}-\mathrm{Cu}$ bonds is observed compared to the refined structure, while the probability of formation of $\mathrm{Zr}-\mathrm{Cu}, \mathrm{Zr}-\mathrm{Ni}$ and $\mathrm{Zr}-\mathrm{Al}$ bonds increases, Figure 5. 
In addition, in the bond angle distributions for both glasses, Figure 6, the probability to find angles at about 60 and 120 degrees increases in the energy-optimised models, while the probability of the angles to be at about 90 degrees decreases notably. This may indicate that fragments of hexagonal or body centered type packing are energetically preferred in local coordination polyhedra to fragments of face centered or cubic type close packing. The improved energetic stability appears to increase the probability of finding a dihedral angle around 40, 80 and 150 degrees for the energy optimized models as compared to the randomly packed or refined models, Figure 7.

In general, however, there is a considerable degree of randomness in the packing of the studied metallic glass, as models with the bond type, bond angle and dihedral angle distributions close to those observed in the randomly packed models fit the diffraction data well. According to both the DFT-energy optimized model and the randomly packed model of the Zr64CuNiAl glass, $\mathrm{Zr}-\mathrm{Zr}$ bonding predominates, while with increase in the $\mathrm{Cu}$ content in the $\mathrm{Zr} 55 \mathrm{CuNiAl}$ glass, the number of $\mathrm{Zr}-\mathrm{Zr}$ bonds decreases while the number of $\mathrm{Zr}-\mathrm{Cu}$ bonds increases. This can explain the shortening of the first neighbour distances in the $\mathrm{Zr} 55 \mathrm{CuNiAl}$ glass, as longer $\mathrm{Zr}-\mathrm{Zr}$ are replaced by shorter $\mathrm{Zr}-\mathrm{Cu}$ bonds.

A randomly packed structure of the studied glasses with a small degree of local ordering suggested in the present study agrees with a previous investigation of enthalpy of mixing in $\mathrm{Zr}$ $\mathrm{Cu}-\mathrm{Al}$ and $\mathrm{Zr}-\mathrm{Cu}-\mathrm{Ni}-\mathrm{Al}$ alloys [20]. In that study, using an association model with various binary and ternary stable associates representing local ordering, good agreement of the experimental enthalpy of mixing and temperature dependence of the heat capacity of the alloys with corresponding model parameters was obtained. It was, however, shown that the molar contributions of the associates were very small [20]. 
We note that the energy-optimized structures discussed here were arrived at starting from randomly packed models. It may therefore happen that this is just one of the possible energetically stable models of the material, and other energetically stable models may also be feasible. To investigate this, extensive reliable molecular dynamics simulations of the systems may be needed, which are a goal of future work.

It has been suggested that $\mathrm{ZrCu}(\mathrm{Ni}) \mathrm{Al}$ metallic glasses with more densely packed local coordination polyhedra (having higher average coordination numbers) are less plastic, with $\mathrm{Zr}_{64} \mathrm{Cu}_{26} \mathrm{Al}_{10}$ being more plastic than $\mathrm{Zr}_{45} \mathrm{Cu}_{45} \mathrm{Al}_{10}$ [14]. The variation of the coordination number with composition agrees well with the results of the present structural study for the $\mathrm{ZrCuNiAl}$ glasses. The coordination numbers of both $\mathrm{Cu}$ and $\mathrm{Al}$ are higher in the $\mathrm{Zr} 55 \mathrm{CuNiAl}$ than those in the $\mathrm{Zr64CuNiAl}$ metallic glass, while coordination numbers of $\mathrm{Zr}$ and $\mathrm{Ni}$ are the same.

\section{Conclusions}

Refined structures for both studied glasses have indicated a considerable similarity with the corresponding randomly packed models. Although the DFT calculations have suggested the presence of some local ordering, several of the main bond lengths from the RMC refinements disagree considerably with those computed in the DFT energy optimizations. Local ordering reflected in the formation of preferred bond angles and dihedral angles in the material found in the DFT calculations, however, still cannot be excluded. The largely random structure of the glasses with a degree of local ordering agrees with previous thermodynamic studies of similar systems and can be important in understanding the unusually high plasticity of the materials. 


\section{Acknowledgements}

We thank Professor Y. Zhang (University of Science and Technology, Beijing) for the provision of samples of the metallic glasses. KBB was supported by the EPSRC Grant (EP/F048009/1).

\section{References}

[1] D. J. H. Cockayne and D.R. McKenzie, Acta Cryst. A 44 (1988) p.870.

[2] D. J. H. Cockayne, D. R. McKenzie and D. Muller, Microanalysis, Microscopy, Microstructure 2 (1991) p. 359.

[3] D. J. H. Cockayne, Annu. Rev. Mater. Res. 37 (2007) p.159.

[4] D. A. Keen and R. L. McGreevy, Nature 344 (1990) p.423.

[5] R. L. McGreevy, J. Phys. Condens. Matter 13 (2001) p.R877.

[6] Y. Idota, T. Kubota, A. Matsufuji, Y. Maekawa and T. Miyasaka, Science 276 (1997) p.1395.

[7] L. Greer, Science 267 (1995) p.1947.

[8] Y. Liu, G. Wang, R. Wang, D. Zhao, M. Pan and W. Wang, Science 315 (2007) p.1385.

[9] J. Das, M. B. Tang, K. B. Kim, R. Theissmann, F. Baier, W. H. Wang and J. Eckert, Phys. Rev. Lett. 94 (2005) p. 205501.

[10] S. Zhu, X. Wang, F. Qin and A. Inoue, Mater. Trans. 48 (2007) p.1748.

[11] K. Georgarakis, A. R. Yavari, D. V. Louzguine-Luzgin, J. Antonowicz, M. Stoica, Y. Li, M. Satta, A. LeMoulec, G. Vaughan, and A. Inoue, Appl. Phys. Lett. 94 (2009) p.191912.

[12] J. Antonowicz, D.V. Louzguine-Luzgin, A.R. Yavari, K. Georgarakis, M. Stoica, G. Vaughan, E. Matsubara and A. Inoue, J. Alloys and Comp. 471 (2009) p.70. 
Page 13 of 30

Philosophical Magazine \& Philosophical Magazine Letters

$$
\begin{aligned}
& 1 \\
& 2 \\
& 3 \\
& 4 \\
& 5 \\
& 6 \\
& 7 \\
& 8 \\
& 9 \\
& 10 \\
& 11 \\
& 12 \\
& 13 \\
& 14 \\
& 15 \\
& 16 \\
& 17 \\
& 18 \\
& 19 \\
& 20 \\
& 21 \\
& 22 \\
& 23 \\
& 24 \\
& 25 \\
& 26 \\
& 27 \\
& 28 \\
& 29 \\
& 30 \\
& 3
\end{aligned}
$$

[13] Y. Q. Cheng, E. Ma and H.W. Sheng, Phys. Rev. Lett. 102 (2009) p.245501.

[14] L. Zhang, Y.-Q. Cheng, A-J. Cao, J. Xu and E. Ma, Acta Materialia 57 (2009) p.1154.

[15] E. J. Kirkland, Advanced computing in electron microscopy. Plenum Press; 1998. p.202.

[16] G. Kresse and J. Hafner, J. Phys Rev B 48 (1993) p.13115.

[17] G. Kresse and J. Hafner, Phys Rev B 49 (1994) p.14251.

[18] J. P. Perdew and A. Zunger, Phys Rev B 23 (1981) p.5048.

[19] H. J. Monkhorst, J. D. Pack, Phys. Rev. B 13 (1976) p.5188.

[20] S. H. Zhou, J. Schmid and F. Sommer, Thermochim. Acta 339 (1999) p.1.

http://mc.manuscriptcentral.com/pm-pml 
Table 1. Peak positions $(\AA)$ in the experimental RDFs of $\mathrm{Zr64CuNiAl}$ and $\mathrm{Zr} 55 \mathrm{CuNiAl}$ metallic glasses as compared with the RDFs from DFT calculations

\begin{tabular}{|c|c|c|c|c|}
\hline Peak & Zr64CuNiAl & & $\mathrm{Zr} 55 \mathrm{CuNiAl}$ & \\
\hline & $\operatorname{Exp}( \pm 0.02 \AA)$ & DFT & $\operatorname{Exp}( \pm 0.02 \AA)$ & DFT \\
\hline 1 & 3.18 & 3.04 & 3.06 & 2.94 \\
\hline 2 & 5.64 & 5.40 & 5.51 & 5.12 \\
\hline 3 & 8.13 & - & 7.91 & - \\
\hline 4 & 10.80 & - & 10.75 & - \\
\hline
\end{tabular}


Table 2. Main average bond distances $(\AA)$ and coordination numbers from the DFT energy optimisations as compared to those obtained in the constrained RMC refinements for Zr64CuNiAl and Zr55CuNiAl metallic glasses

\begin{tabular}{|c|c|c|c|c|}
\hline Parameter & Zr64CuNiAl & & $\mathrm{Zr} 55 \mathrm{CuNiAl}$ & \\
\hline & DFT & $\begin{array}{l}\text { RMC } \\
( \pm 0.02 \AA)\end{array}$ & DFT & $\begin{array}{l}\text { RMC } \\
( \pm 0.02 \AA)\end{array}$ \\
\hline $\mathrm{Zr}-\mathrm{Zr}$ & 3.17 & 3.13 & 3.18 & 3.12 \\
\hline $\mathrm{Zr}-\mathrm{Cu}$ & 2.84 & 2.96 & 2.89 & 2.96 \\
\hline $\mathrm{Zr}-\mathrm{Ni}$ & 2.74 & 2.69 & 2.78 & 2.71 \\
\hline $\mathrm{Zr}-\mathrm{Al}$ & 2.98 & 2.67 & 2.99 & 2.65 \\
\hline $\mathrm{Cu}-\mathrm{Cu}$ & 2.67 & 2.78 & 2.61 & 2.80 \\
\hline $\mathrm{Cu}-\mathrm{Ni}$ & 2.61 & 2.52 & 2.59 & 2.54 \\
\hline $\mathrm{Cu}-\mathrm{Al}$ & 2.53 & 2.52 & 2.63 & 2.52 \\
\hline$N_{\mathrm{Zr}}$ & 9.8 & 7.8 & 9.8 & 7.8 \\
\hline$N_{\mathrm{Cu}}$ & 8.7 & 7.0 & 9.1 & 7.4 \\
\hline$N_{\mathrm{Ni}}$ & 8.2 & 5.8 & 8.5 & 5.8 \\
\hline$N_{\mathrm{Al}}$ & 8.0 & 6.2 & 8.5 & 6.5 \\
\hline
\end{tabular}


Figure captions

Figure 1. Comparison of the experimental RDFs from the two metallic glasses.

Figure 2. Comparison of the experimental (Exp) RDFs with those obtained from the energyoptimised small model (DFT) for a) Zr64CuNiAl and b) Zr55CuNiAl glasses.

Figure 3. Comparison of the experimental (Exp) RDFs with those obtained from randomly packed models for a) $\mathrm{Zr64CuNiAl}$ and b) $\mathrm{Zr} 55 \mathrm{CuNiAl}$ glasses.

Figure 4. Comparison of the experimental (Exp) RDFs with those obtained from RMC refinements (RMC) for a) $\mathrm{Zr64CuNiAl}$ and b) $\mathrm{Zr} 55 \mathrm{CuNiAl}$ glasses.

Figure 5. Normalised bond type distribution histograms from refined models (RMC) as compared to the energy optimized small model (DFT) and randomly packed model for a) $\mathrm{Zr64CuNiAl}$ and b) Zr55CuNiAl glasses.

Figure 6. Normalised bond angle distribution histograms from refined models (RMC) as compared to the energy optimized small model (DFT) and randomly packed model for a) Zr64CuNiAl and b) Zr55CuNiAl glasses. 
Figure 7. Normalised dihedral angle distribution histograms from refined models (RMC) as compared to the energy optimized small model (DFT) and randomly packed model for a) Zr64CuNiAl and b) Zr55CuNiAl glasses.

1

2

3

4

5

6

7

8

9

10

11

12

13

14

15

16

17

18

19

20

21

22

23

24

25

26

27

28

29

30

31

32

33

34

35

36

37

38

39

40

41

42

43

44

45

46

47

48

49

50

51

52

53

54

55

56

57

58

59

60 


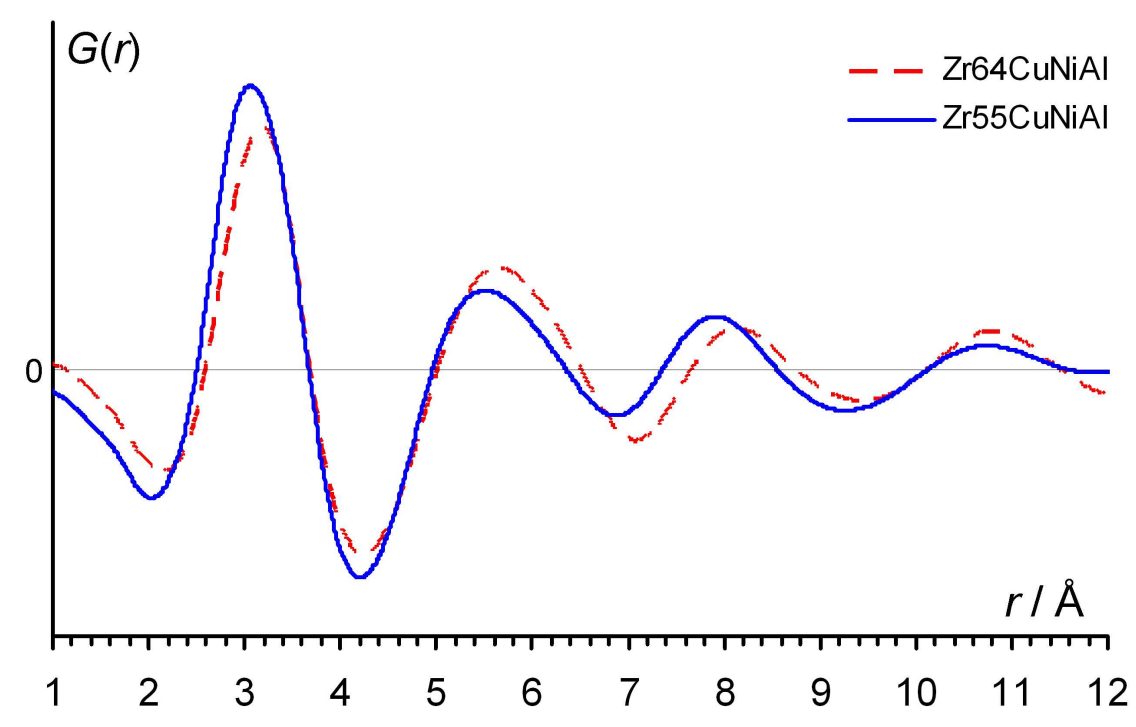

$254 \times 190 \mathrm{~mm}(300 \times 300 \mathrm{DPI})$ 


1
2
3
4
5
6
7
8
9
10
11
12
13
14
15
16
17
18
19
20
21
22
23
24
25
26
27
28
29
30
31
32
33
34
35
36
37
38
39
40
41
42
43
40
45
49
50
51
52
53
55
50

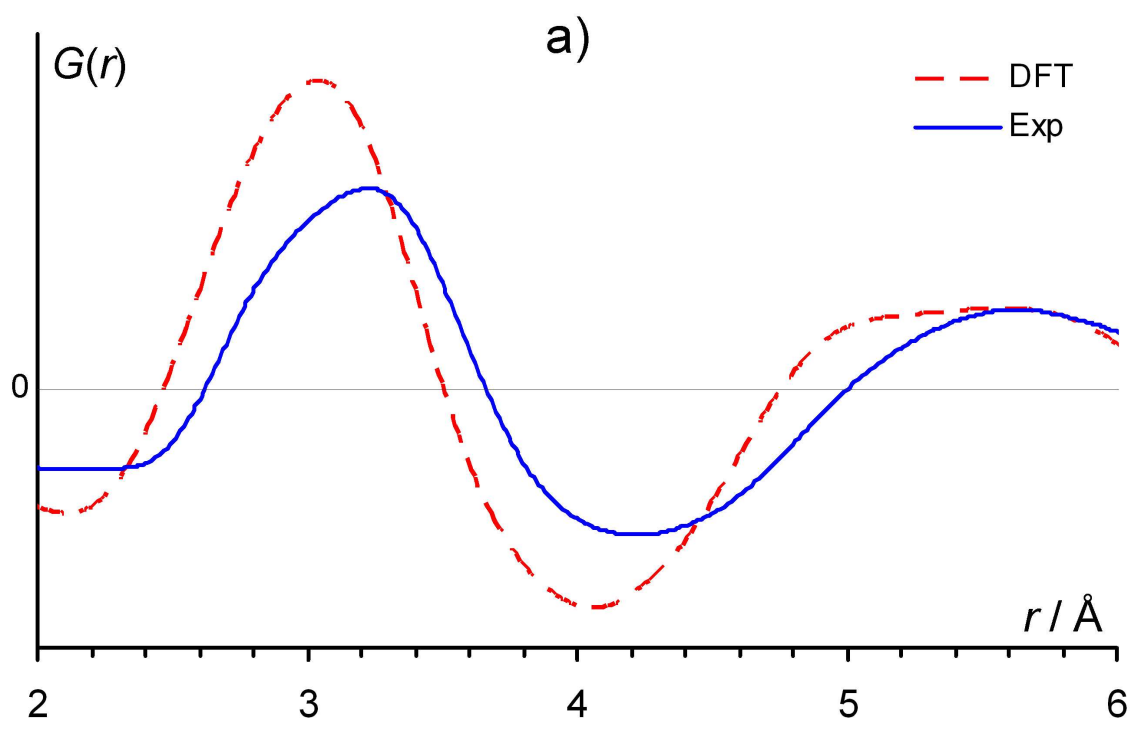

$254 \times 190 \mathrm{~mm}(300 \times 300 \mathrm{DPI})$ 


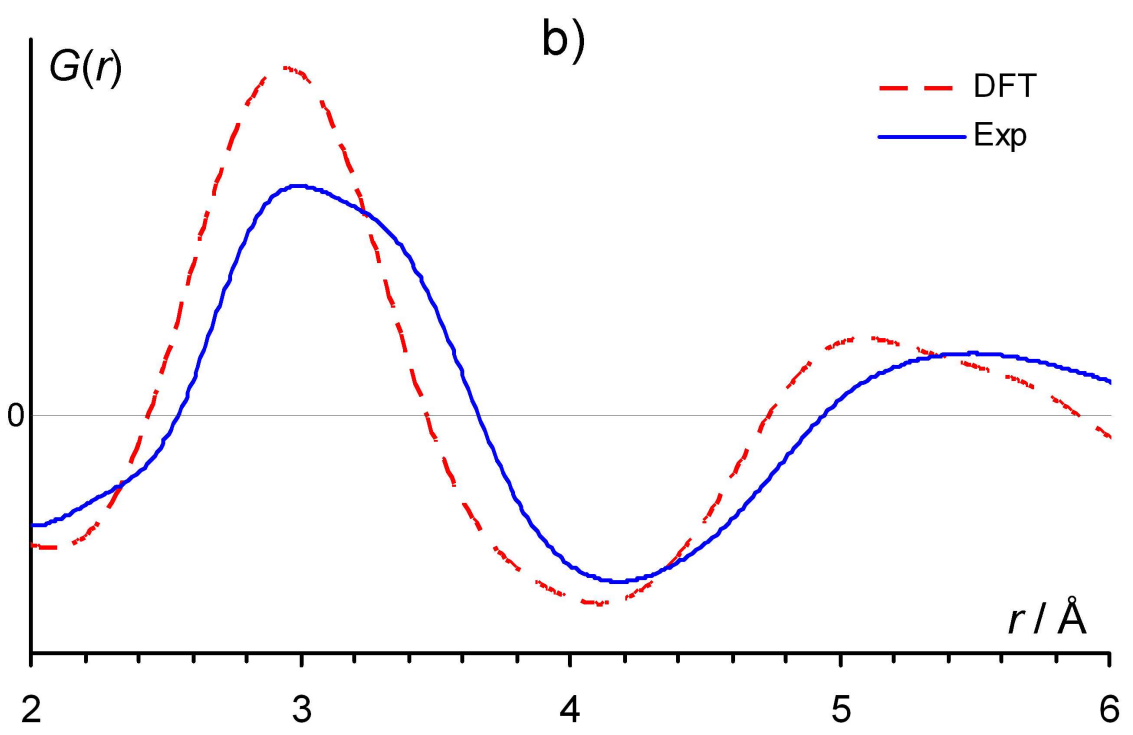

$254 \times 190 \mathrm{~mm}(300 \times 300 \mathrm{DPI})$ 


1
2
3
4
5
6
7
8
9
10
11
12
13
14
15
16
17
18
19
20
21
22
23
24
25
26
27
28
29
30
31
32
33
34
35
36
37
38
39
40
41
42
43
44
45
46
47
48
49
50
51
52
53
54
55
56
57
58
60

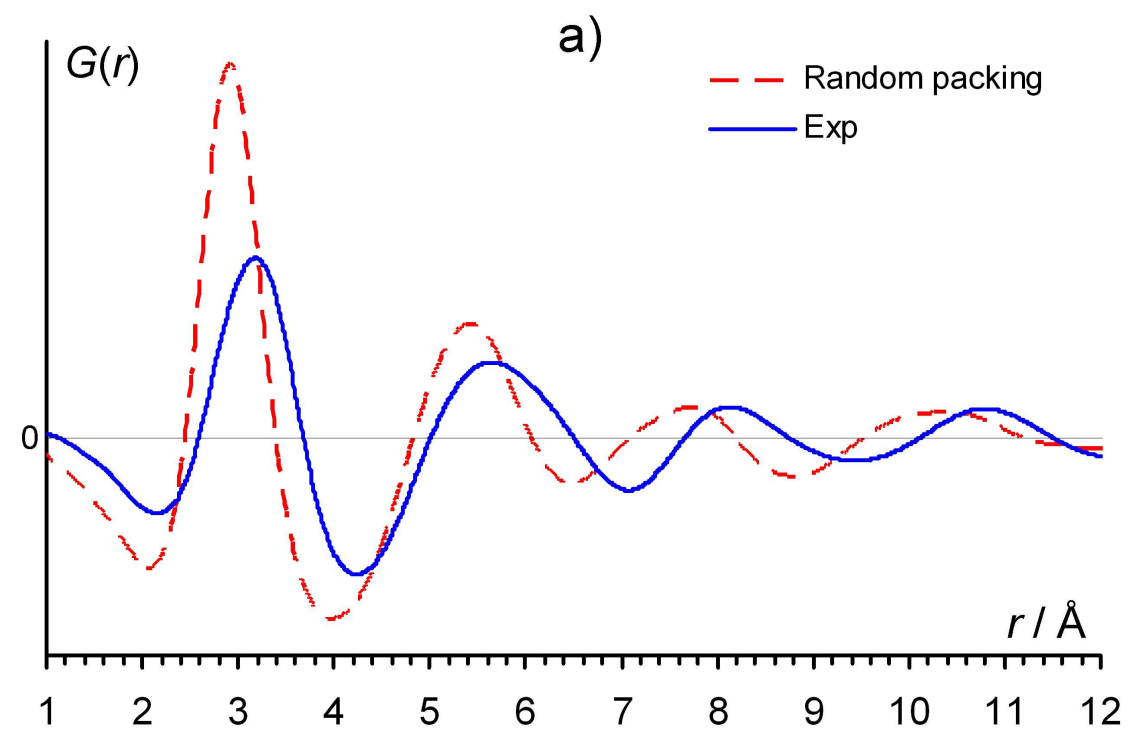

$254 \times 190 \mathrm{~mm}(300 \times 300$ DPI $)$ 


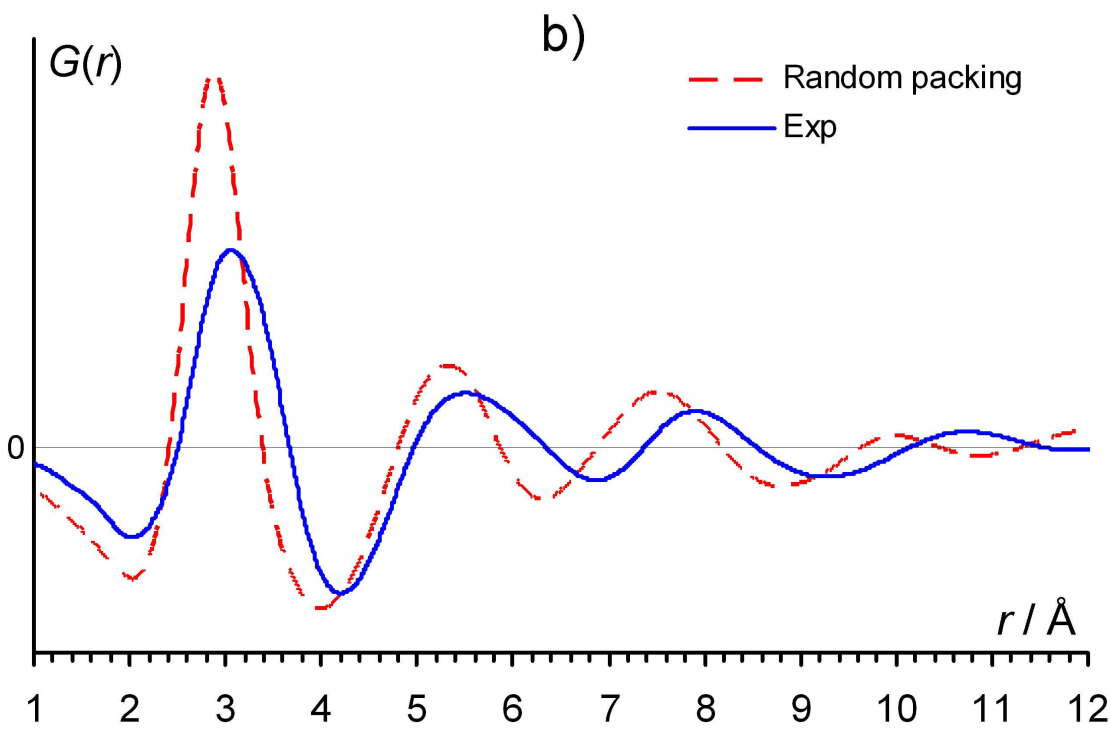

$254 \times 190 \mathrm{~mm}(300 \times 300 \mathrm{DPI})$ 


1
2
3
4
5
6
7
8
9
10
11
12
13
14
15
16
17
18
19
20
21
22
23
24
25
26
27
28
29
30
31
32
33
34
35
36
37
38
39
40
41
42
43
44
45
46
47
48
49
50
51
52
53
54
55
56
57
58
60

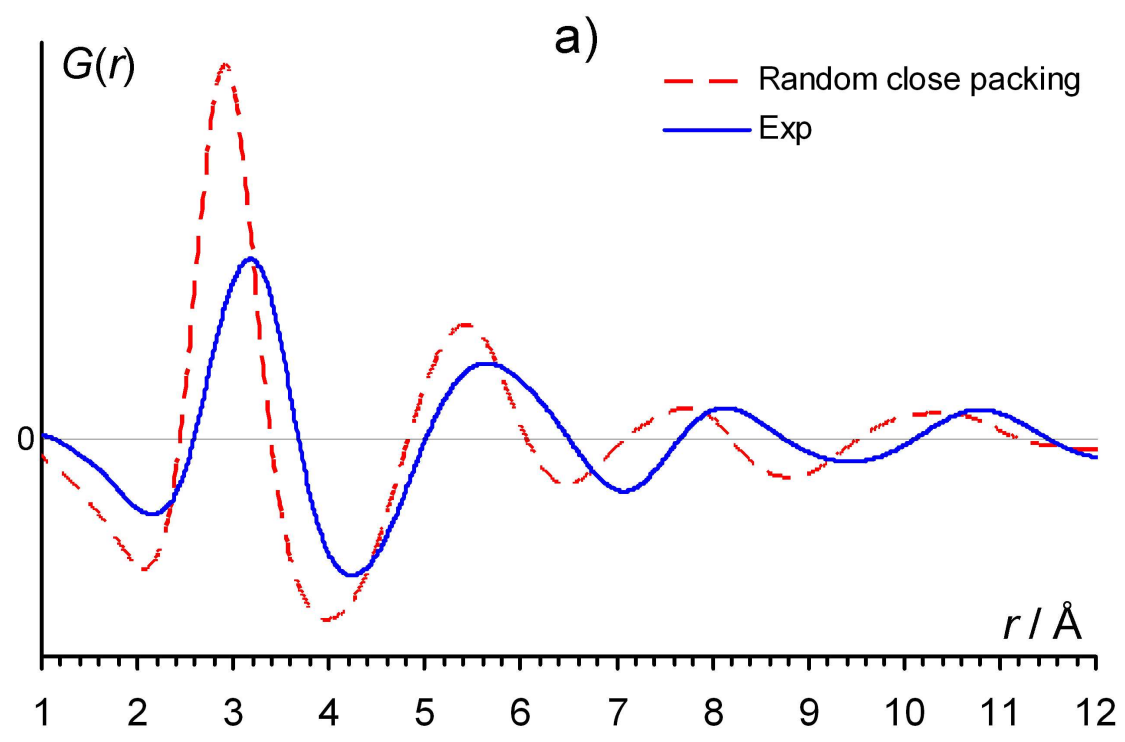

$254 \times 190 \mathrm{~mm}(300 \times 300 \mathrm{DPI})$ 


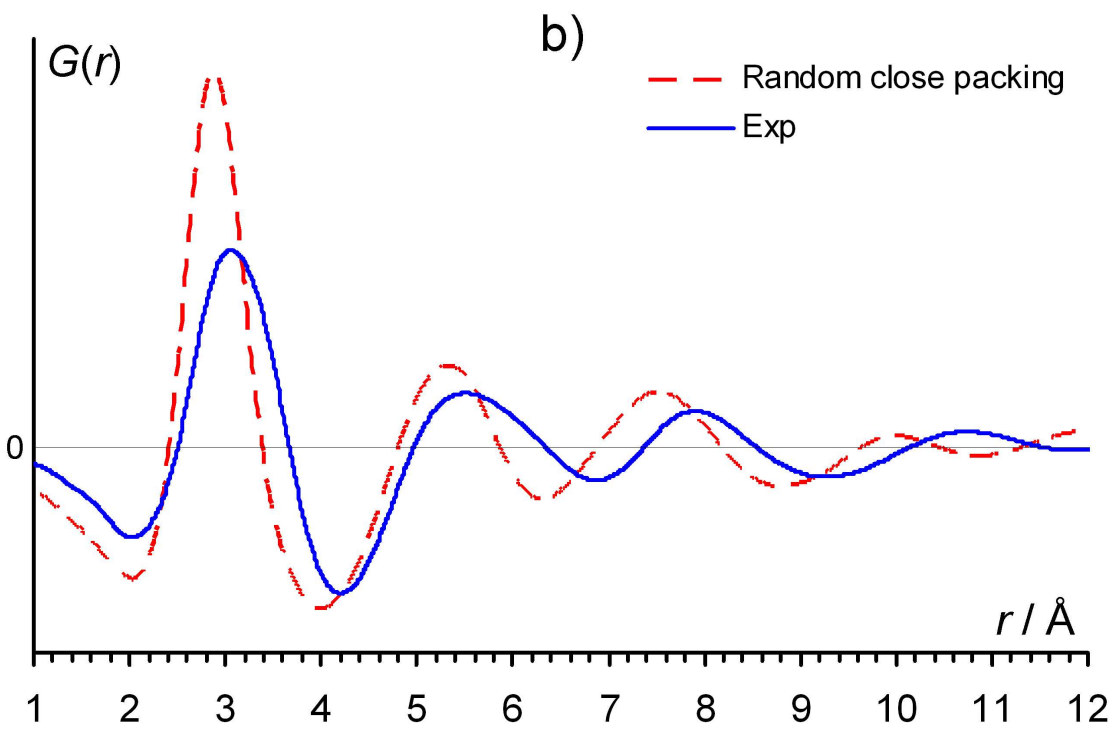

$254 \times 190 \mathrm{~mm}(300 \times 300 \mathrm{DPI})$ 


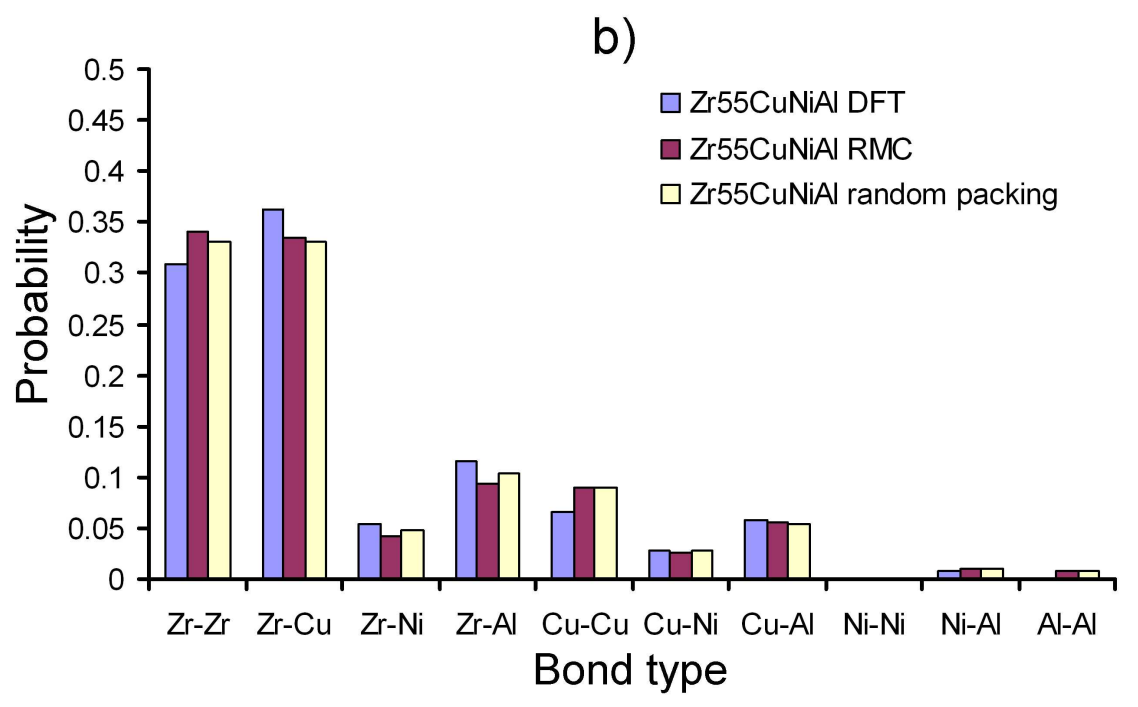

$254 \times 190 \mathrm{~mm}(300 \times 300$ DPI $)$ 


1
2
3
4
5
6
7
8
9
10
11
12
13
14
15
16
17
18
19
20
21
22
23
24
25
26
27
28
29
30
31
32
33
34
35
36
37
38
39
40
41
42
43
44
45
46
47
48
49
50
51
52
53
54
55
56
57
58
60

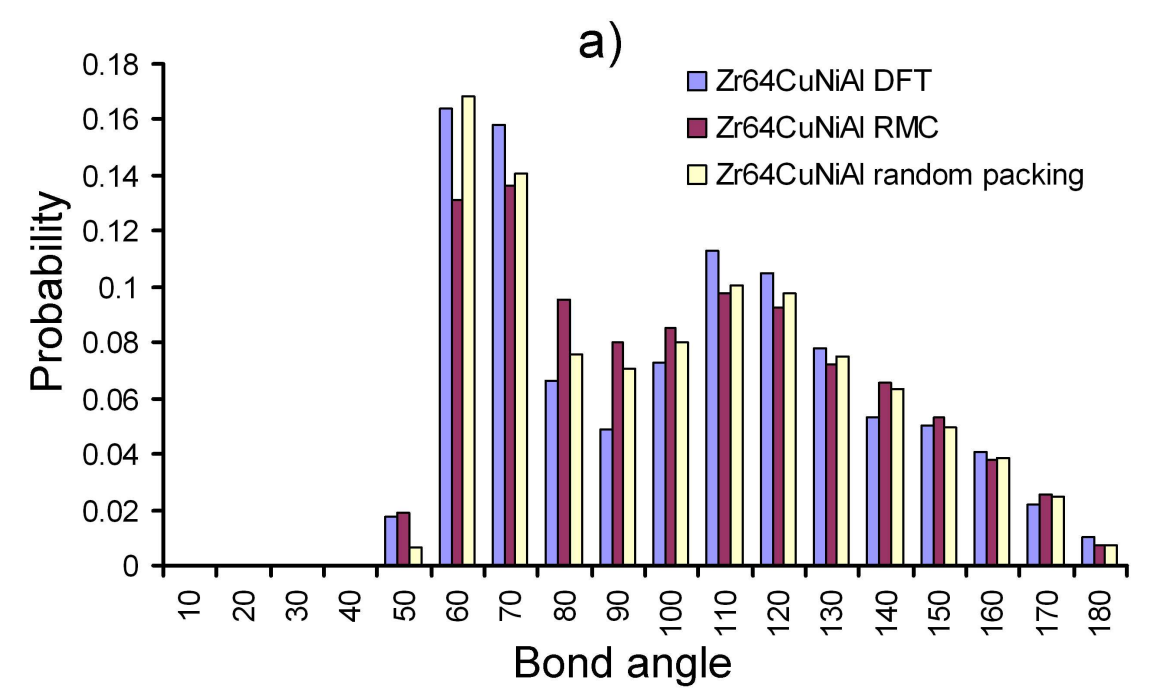

$254 \times 190 \mathrm{~mm}(300 \times 300 \mathrm{DPI})$ 
b)

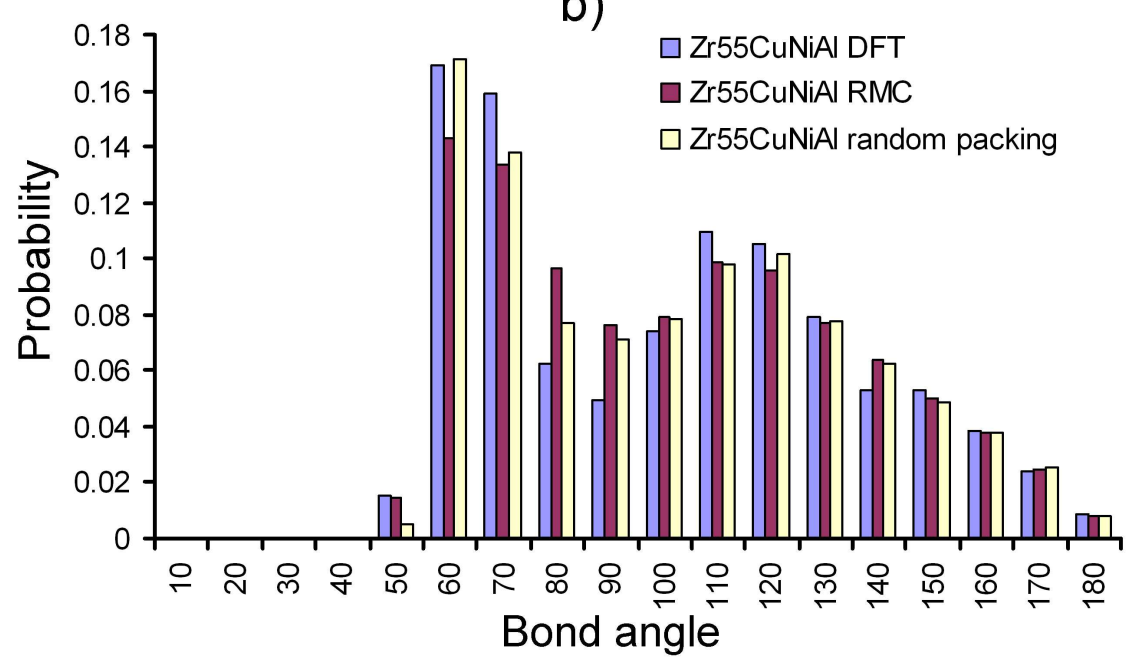

$254 \times 190 \mathrm{~mm}(300 \times 300 \mathrm{DPI})$ 


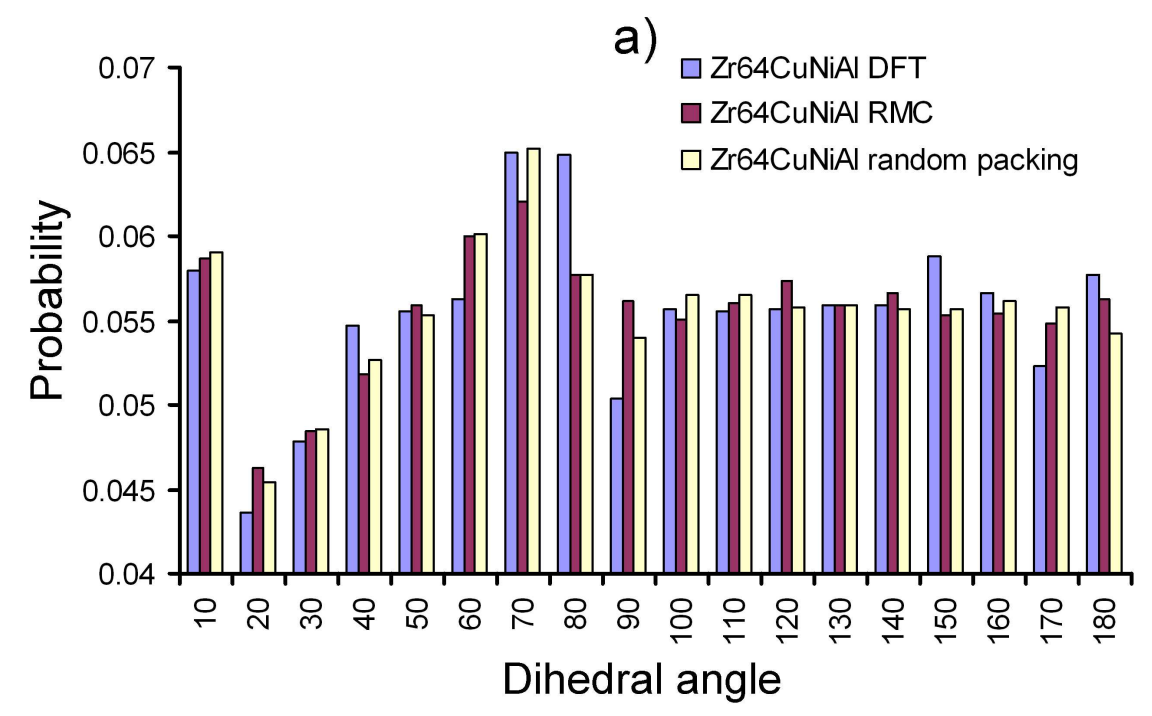

$254 \times 190 \mathrm{~mm}(300 \times 300 \mathrm{DPI})$ 


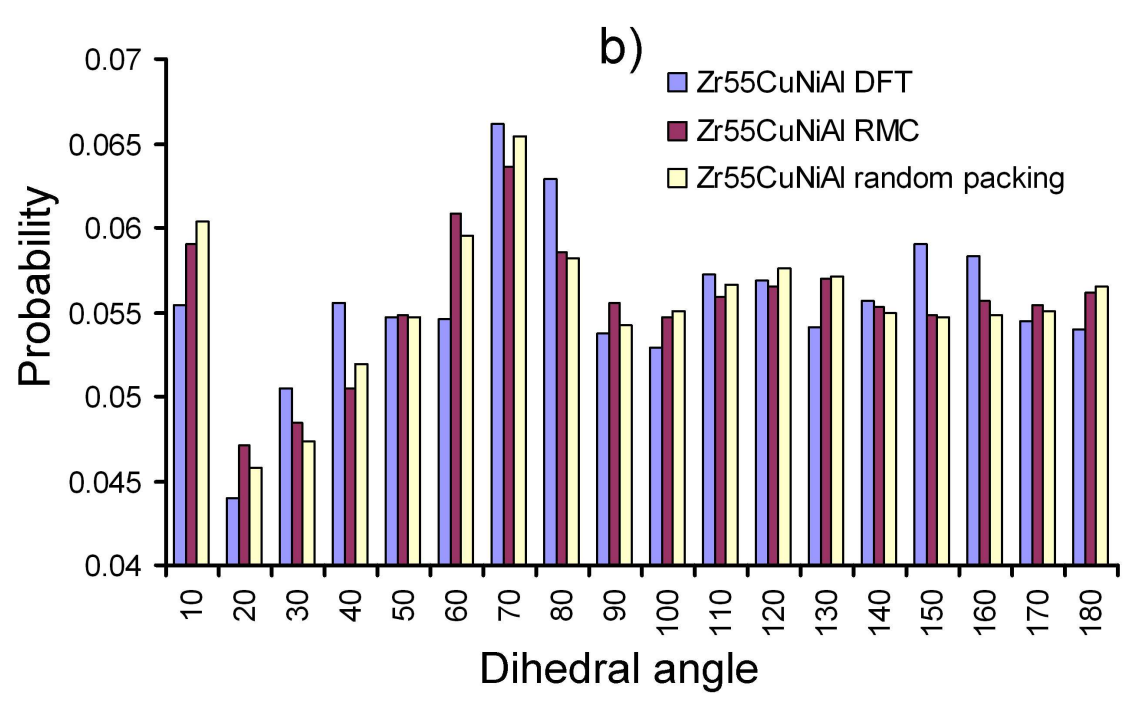

$254 \times 190 \mathrm{~mm}(300 \times 300 \mathrm{DPI})$ 